\title{
ESTRATEGIAS EN INTERNET A PARTIR DE UN MODELO DE DINÁMICA COMPETITIVA
}

Juan Carlos García Villalobos

Ruth Mateos de Cabo

Luis López Fernández

Migue Ángel Fernández San Juan

Universidad CEU San Pablo

Universidad Rey Juan Carlos

garvil@ceu.es

matcab@ceu.es

https://doi.org/10.17979/redma.2009.01.01.4673

\section{Cv Juan Carlos García Villalobos}

Profesor Agregado de Comercialización e Investigación de Mercados (Departamento de Empresa)

Facultad de Ciencias Económicas y Empresariales

Universidad CEU San Pablo

C/ Julián Romea 23. 28003-Madrid

Tel: 91 514.01.16. Fax.: 91 553. 94.85

\section{Cv Ruth Mateos de Cabo}

Profesora Adjunta de Comercialización e Investigación de Mercados (Departamento de Empresa)

Facultad de Ciencias Económicas y Empresariales

Universidad CEU San Pablo

C/ Julián Romea 23. 28003-Madrid

(Grupo de Análisis de Dinámica Económica Caótica)

Tel: 91 456.63.00. Fax.: 91 554.84.96

\section{Luis López Fernández}

Profesor Ayudante Doctor del Departamento Informática, Estadística y Telemática

(Laboratorio de Algoritmia Distribuida y Redes)

Universidad Rey Juan Carlos

C/ Tulipán s/n. 28933-Móstoles (Madrid)

\section{Miguel Ángel Fernández Sanjuán}

Catedrático del Departamento de Ciencias Experimentales e Ingeniería. Responsable del área de Física

(Grupo de Caos y Dinámica No Lineal)

Universidad Rey Juan Carlos

C/ Tulipán s/n. 28933-Móstoles (Madrid) 


\section{Resumen}

En el presente trabajo se estudia un modelo que describe las principales características del mercado de Internet y las dinámicas competitivas de los denominados sitios web. Este modelo, basado en las ecuaciones de LotkaVolterra, analiza situaciones del tipo 'el ganador se lleva todo' manifestadas recurrentemente en mercados altamente competitivos.

Con base en su tratamiento tanto cuantitativo como cualitativo se ofrecen una serie de recomendaciones estratégicas para mejorar resultados, por supuesto, coherentes con las tendencias observadas en la realidad de la Red. Dentro de estas recomendaciones destaca la estrategia de coopetición, que se propone como una opción viable para que los sitios pequeños puedan hacer frente a los grandes líderes de Internet; reforzada por su fundamento en consonancia con las predicciones propias de un modelo de dinámica competitiva.

Junto a ello, también se formulan diversas estrategias para los sitios de nueva creación o recién llegados al mercado (los conocidos start-ups) como la de segmentación y los programas de filiación entre portales verticales.

\section{Palabras clave:}

Internet; dinámica competitiva; ecuaciones diferenciales; comportamiento estratégico.

\section{Abstract \\ INTERNET STRATEGIES BASED ON A DYNAMIC COMPETITIVE MODEL}

The present paper studies the characteristics and strategies suggested by a model of web sites competition dynamics, based on the Lotka-Volterra competition equations. The model captures the well know winner-take-all characteristic that is caused by the strong positive feedback mechanisms linked to high competence. 
From the analysis, both quantitative and qualitatively, of the model a series of strategic recommendations are offered to reach success, that prove to be coherent with the trends observed in the Internet real market. Among the above mentioned recommendations, the strategy of coopetition is highlighted as the most suitable option for small sites in order to deal with the leaders of the Net, being this one of the most interesting and novel behaviors that arise from the above mentioned analysis. This alternative strategy to the competition is backed by the predictions offered by a model of competitive dynamics.

Furthermore, a series of interesting strategies for start-ups sites or new comers to the market are suggested, like segmentation and affiliation programs between vertical portals.

Códigos JEL: C68; L86

\section{Keywords:}

Internet, competition dynamics, differential equations, strategic behaviour. 


\section{INTRODUCCIÓN}

Mientras que para algunos Internet no es más que una herramienta para transportar bits de información, para otros es una importante fuerza de cambio que tiende a alterar la estructura de los sectores e incluso los paradigmas bajos los cuales los participantes operan en los mercados.

Bajo el impulso de esta nueva infraestructura se ha pasado de una Economía Industrial y material a una Economía Postindustrial conocida como Era de la Información (Nieto de Alba, 1998). En este escenario, la cuestión para los participantes no es tanto la de aplicar o no Internet, sino cómo utilizarla para conseguir la supervivencia o incluso el liderazgo.

Las empresas invierten grandes cantidades de recursos intentado proveer servicios, software, equipos y comercio electrónico a través de Internet. McNight et al. (2001) insisten en que para sobrevivir en la Red necesitan algo más que un sitio web, como poseer una tecnología, un bien o servicio que alguien desee obtener. En resumidas cuentas, para conseguir una ventaja competitiva las empresas tratan de crear valor para los clientes (Porter, 2001).

Así, lejos de hacer de los aspectos estratégicos una cuestión menor, Internet convierte la estrategia en un aspecto vital para asegurar el éxito y la supervivencia en un entorno tan turbulento y complejo. Con dicho objeto están surgiendo nuevos modelos y paradigmas en las últimas décadas que intentan contribuir a una mejor comprensión de lo que realmente está sucediendo.

Este artículo parte de una propuesta de un modelo dinámico no lineal como herramienta para el estudio de algunas características relevantes de Internet e incluso de diversos tipos de comportamientos estratégicos. Para ello, se estructura en las siguientes partes: 
1. Análisis de la naturaleza y especificidades de Internet y la adecuación del uso de herramientas de dinámica no lineal, caso del modelo de Lotka-Volterra.

2. Formulación del modelo y de su análisis técnico.

3. Explicación de los tipos de mercado que se pueden presentar en función de los planteamientos previos.

4. Propuesta de recomendaciones estratégicas sugeridas por el modelo según tamaño del sitio web y del tipo de mercado en que opere.

\section{LA NATURALEZA DE INTERNET}

Internet constituye la mayor conexión de diferentes redes de ordenadores que emplean un conjunto de protocolos comunes de uso universal (el Protocolo de Control Transmisión/Protocolo de Internet, TCP/TI) para comunicarse e intercambiar información (Rodríguez Ardura, 2002). En esencia, Internet constituye una plataforma inteligente, ubicua y flexible de información (Sharma, 2002). Gracias a su componente interactivo, las organizaciones utilizan dicha plataforma para proveer información y soporte al cliente, crear un diálogo permanente con él que les permita fortalecer sus relaciones y explorar nuevas oportunidades de venta (Dutta y Biren, 2001).

Características igualmente interesantes de esta poderosa herramienta de transmisión de información son su alta conectividad, ya que constituye un sistema de información instantáneo y permanente y que da lugar a nuevos mecanismos de comunicación y coordinación entre las empresas y sus clientes; y al mismo tiempo, la posibilidad de constituir comunidades virtuales que comparten experiencias en áreas específicas (Sharma, 2002). Es más, si la información es la materia prima del proceso de toma de decisiones y esta se produce y se puede transmitir en cualquier momento desde cualquier lugar a cualquier lugar, se está alcanzando una capacidad de democracia convencional (una persona, un voto) en el establecimiento de los objetivos en las organizaciones a través de la Red (GARCÍA-GUTIÉRREZ et al. 2004). 
La economía de Internet presenta unos rasgos genuinos de los que se derivan implicaciones estratégicas que, en algunos aspectos, podrían considerarse alejadas de la lógica que prevalece en los sectores tradicionales. Para Gual y Ricart (2001) los factores que en mayor o menor medida caracterizan la economía de la información son:

A. La estructura de costes. Los costes de diseminación de la información decrecientes en Internet provocan que el coste marginal de transmisión de la información sea prácticamente nulo. Una vez pagados los altos costes fijos de desarrollo de una página web (que normalmente son costes no recuperables o sunk costs), las posibilidades tecnológicas que ofrece la Red hacen que sea relativamente barato incrementar la capacidad necesaria para hacer frente a un aumento de la demanda (Maurer y Huberman, 2000).

Con este argumento económico se derivan dos extremos estratégicos. En primer lugar, se hace patente la tendencia de los bienes y servicios a convertirse en una commodity, con lo que la estrategia debería encaminarse a disminuir esa tendencia de homogeneización de los productos y servicios (Gual y Ricart, 2001). En segundo lugar, subyace que la teoría del equilibrio competitivo tradicional resulta inaplicable (Maurer y Huberman, 2000). Según esta teoría tanto la oferta como la demanda agregada son función de los precios. Dado que en Internet el precio de una página web es prácticamente nulo, la oferta se ajustará siempre a la demanda, de manera que la única variable cuantitativa a considerar es la demanda agregada; que es precisamente la variable para la cual se estudia la dinámica de los sitios web en función de su crecimiento y capacidad, pero también el grado de competencia existente.

B. La aparición del efecto cierre (lock-in). Tiene lugar cuando el consumidor queda atrapado en un bien o servicio como consecuencia de los altos costes de cambio. Estos costes de cambio pueden originarse como consecuencia de una combinación de factores bien sea la existencia de 
compromisos contractuales, la compra de bienes duraderos (hardware, por ejemplo), la formación específica para una marca (por ejemplo, aprender a utilizar un programa), los programas de fidelización, el coste de búsqueda, etc. No obstante, conviene adelantar una implicación estratégica básica de este efecto: en la lucha por la supremacía tecnológica las empresas apuestan por lo que pretenden que sea un producto o tecnología superior, pero donde la rapidez puede ser más importante que la excelencia (Arthur, 1996).

C. La presencia de retroalimentación positiva resultado de una combinación de economías de escala en la oferta con las economías de escala del lado de la demanda. Estas últimas son, con mucho, más importantes en Internet y en su forma extrema pueden dar lugar a mercados en los que un único vendedor acapare todo.

En esta línea, la fuerte tendencia a la monopolización se ve explicada, al menos en parte, por la estructura de costes en Internet. En efecto, puesto que una vez desarrollado el sitio web la mayoría de los costes son costes enterrados o no recuperables y, además, se puede servir a muchos clientes a costes unitarios casi constantes y sin límites de capacidad (Shapiro y Varian, 1999), la competencia entre los distintos sitios puede llegar a ser muy intensa con el fin de tratar de obtener el máximo volumen de visitantes. El proceso se realimenta provocando que exista un solo ganador motivado porque si dos o más sitios web ofrecen contenidos muy próximos, al final los usuarios se decantarían por uno de ellos en perjuicio del otro actor.

\section{LOS MODELOS DE DINÁMICA COMPETITIVA Y LAS EXTERNALIDADES DE RED}


Las ecuaciones de Lotka-Volterra se han utilizado con frecuencia para simular interacciones entre dos o más poblaciones. De acuerdo con este modelo, la población de una especie crece siguiendo una ley logística en ausencia de otras especies e introduce un término de interacción competitiva según el cual la tasa de crecimiento de una población es negativamente proporcional a la competencia entre las distintas poblaciones (Alligood et al., 1996).

Numerosos autores han demostrado la utilidad de los modelos de competencia entre especies en el terreno de las ciencias sociales y más concretamente en el ámbito organizacional. Guastello (1992) los utiliza en estudios sobre la productividad del trabajo, Hannan y Freeman $(1977,1988)$ en la proliferación de organizaciones, y Peters y Hogwood (1991) los aplican para el estudio de organismos públicos.

Además de su utilidad comprobada mediante muchos estudios, estos modelos de ecología de poblaciones presentan una ventaja adicional derivada del hecho de que permiten abordar el estudio de las organizaciones bajo un enfoque que traslada el interés de la comparación de las estructuras de gestión hacia la dinámica y evolución del entorno y de las organizaciones en juego (Choi, 1997).

La elección del modelo de Lotka-Volterra frente a otros modelos de difusión de innovación como el ampliamente utilizado de Bass (1969) que describe las ventas totales en función de la probabilidad de conversión de los clientes innovadores y de la influencia ejercida por éstos sobre los imitadores se fundamenta especialmente por la introducción de un término de competencia no lineal. Éste permite estudiar la dinámica de interacción entre diversos sitios competidores en Internet y cómo determina la evolución de la estructura del mercado y las correspondientes actuaciones estratégicas.

A pesar de que el modelo inicial de Bass y sus extensiones han sido utilizadas con fines estratégicos y normativos ${ }^{1}$, el modelo de Lotka-Volterra resulta especialmente adecuado para reflejar la dinámica de los sitios web. Esto es así 
porque al incorporar fuertes mecanismos de retroalimentación positiva (positive feedback) se producen circunstancias como 'el ganador se lleva todo' que son fruto de las diversas formas de rendimientos crecientes que muestran las empresas que compiten en Internet.

Bajo condiciones de competencia simétrica el modelo de Lotka-Volterra administra una transición desde una situación de cierto equilibrio a otra en la cual un participante se hace con el mercado. Este cambio tiene su razón de ser en la presencia de fuertes mecanismos de retroalimentación positivos que se manifiestan en forma de externalidades de red; o dicho de otro modo, economías de escala del lado de la demanda.

Según Katz y Shapiro (2001), las externalidades de red son un tipo de rendimientos crecientes que muestran algunos productos o tecnologías y que se producen cuando la utilidad que obtienen sus consumidores crece con el número de agentes que los consumen. Estos autores destacan como posibles causas de las externalidades la mejora directa de la calidad del producto al incrementar el número de clientes (por ejemplo, el teléfono de un usuario tiene más valor cuantos más teléfonos estén conectados a la red telefónica), los efectos indirectos derivados del incremento de usuarios (caso de la mejora en la calidad y variedad del software y hardware para los clientes de ordenadores personales similares). Asimismo, para bienes duraderos las externalidades aparecen también cuando la calidad y disponibilidad del servicio postventa depende de la experiencia y el tamaño de la red de asistencia como ocurre en el sector de la automoción.

La presencia de estas externalidades o efectos de red puede dar lugar a que en Internet un sitio eventualmente llegue a marginar a los otros participantes del mercado hasta quedarse con todo convirtiéndose en líder indiscutible (tómese como referencia de sitios web en los que aparecen fuertes efectos de Red el caso de los motores de búsqueda, en particular Google que superó a sus rivales Altavista o alltheweb.com convirtiéndose en el nodo más grande y en el motor de búsqueda más visitado). 
Junto con el tamaño, las externalidades de red pueden desencadenarse por otros mecanismos que tienen como máximo exponente el momento en que una determinada tecnología entra en el mercado. Esto permite identificar el denominado efecto lock-in o cierre, que explica que con frecuencia el cliente pueda estar obligado a un alto coste de cambio de proveedor por una combinación de factores (activos específicos complementarios, formación especial para un sitio, coste de búsqueda, programas de fidelización, etc.). A resultas el cliente puede quedar atrapado en un producto o servicio; de modo que en su lucha competitiva los sitios web apuestan por lo aspiran que sea una tecnología superior, aun cuando la rapidez y el manejo adecuado de la base instalada de clientes se transforman en variables estratégicas fundamentales (Gual y Ricart, 2001). Arthur (1989) ofrece dos ejemplos de tecnologías que establecidas de manera temprana llegaron a convertirse en dominantes a pesar de la superioridad técnica de sus rivales: los reactores de agua ligera en la industria nuclear norteamericana y la competencia entre coches de gasolina y vapor. Sin embargo, la demostración de un sitio que entró en la Red lo suficientemente pronto como para ganar una cuota de mercado que le proporcionase una posición de manifiesta superioridad inicial frente a sus competidores fue el portal Amazon. De hecho, desde hace tiempo se le considera el detallista on-line más importante del mundo, lo que es reconocido por los inversores a través de un alto valor para sus acciones pese a que durante mucho tiempo no se materializó en rentabilidad (Ohmae, 2000).

No obstante, no todo son ventajas en la condición de pionero del mercado (liderazgo tecnológico, anticipación en la compra de activos escasos y desarrollo de costes de cambio para el cliente), ya que la preponderancia de las desventajas puede anularlas y en el caso extremo provocar que finalmente sea seguidor el a priori ganador. En este sentido, Lieberman y Montgomery (1988) destacan, entre otros, el efecto free-rider (polizón), la resolución tecnológica o de la incertidumbre del mercado, los cambios en la tecnología o en las necesidades de los consumidores y diversos tipos de inercia 
organizativa (esto sucedió con Google en el segmento de los motores de búsqueda como se ha mencionado más arriba)

En otro orden, los estudios empíricos de Shankar y Bayus (2003) en el mercado de las videoconsolas muestran que la presencia de los efectos de red son función tanto del tamaño de la red de clientes de un negocio como de la fuerza de dicha red. De esta forma, estos efectos en situación de simetría entre los participantes ocasionan que con fuerte competencia (que para esos autores se traduce en incompatibilidad entre tecnologías) la empresa con la base de clientes más amplia tenga ventaja sobre sus competidores y llegue a liderar el mercado. Ahora bien, cuando las simetrías desaparecen y dejan paso a la interacción de la fuerza de la red de clientes con variables de administración empresarial (precio o publicidad), el resultado es que empresas con una número de clientes relativamente bajo puede sobrevivir con éxito junto a los líderes del mercado.

Si bien los efectos de red no aparecen explícitamente en las ecuaciones de Lotka-Volterra, el modelo muestra su presencia al producirse, en condiciones que han sido ampliamente descritas por Maurer y Huberman (2000), el fenómeno del 'ganador se lleva todo' el mercado. También evidencia bajo condiciones más generales otros tipos de comportamiento y estrategias propias de los mercados mixtos en los que algunos competidores colaboran mientras mantienen una intensa competencia con el resto de los participantes. En este escenario se proponen estrategias alternativas a la mera competición para la supervivencia frente a los grandes de Internet, en concreto la coopetición y la segmentación, tal y como se explica más abajo.

\section{EL MODELO Y SU ANÁLISIS}

Cualquier mercado es lo suficientemente complejo como para que nunca sea explicado completamente a través de modelos matemáticos, aunque hay algunos de menor complejidad específicos de ciertos segmentos del mercado que incorporan las principales características del comportamiento en Internet REDMARKA - Revista Académica de Marketing Aplicado - CIECID - Universidad de A Coruña Año I, Número 1, (2009), pp. 39-82 
(López y Sanjuán, 2001). El modelo de partida del estudio se basa en el propuesto por Maurer y Huberman (2000). La razón de su elección es, entre otras, su buen comportamiento en el proceso de obtención de la información sobre las características del mercado en Internet.

Concretamente, se consideran $n$ sitios web que ofrecen servicios similares y compiten por la misma población de usuarios, considerada muy superior al número de sitios. Cada sitio toma parte en programas destinados a incrementar su tasa de clientes base $\left(f_{i}\right)$ que van desde la realización de campañas publicitarias hasta promociones basadas en los precios. Inicialmente el modelo se formuló considerando $f_{i}$ como la parte de la población conocedora del sitio en cuestión (López y Sanjuán, (2001) extienden su definición para que represente su fracción de clientes). En esta línea, se presupone que un mismo usuario puede ser cliente al mismo tiempo de todos los sitios, con lo puede darse el caso de segmentos donde el cien por cien del mercado frecuente todos los sitios.

La evolución temporal de la fracción de clientes $f_{i}$ de un sitio dado $i$ viene determinada por dos factores fundamentales (Maurer y Huberman, 2000). Si no hay competencia con otros sitios, la base de usuarios crece inicialmente de forma exponencial a una tasa $\alpha_{i}$ y se satura a un valor $\beta_{i}$. Estos valores vienen definidos por la capacidad de un sitio para atender a un número dado de visitantes por unidad de tiempo. Si otros sitios ofrecen servicios competitivos la competencia condiciona que el usuario visite varios sitios competidores (niveles de competencia bajos) o que el hecho de haber visitado un sitio reduzca la probabilidad de visitar otro (niveles de competencia altos).

En estos términos la competencia puede entenderse del siguiente modo: si las fracciones $f_{i}$ y $f_{j}$ de gente utiliza los sitios $i$ y $j$ respectivamente, entonces, bajo el supuesto de que la probabilidad de visitar un sitio es independiente de la de usar otro, la fracción $f_{i} f_{j}$ será la de usuarios de ambos sitios. Pero si ambos sitios proporcionan servicios similares algunos de esos usuarios dejarán de 
utilizar uno u otro sitio. La tasa a la que cesarán de utilizar el sitio $i$ será $\gamma_{i j} f_{i} f_{j}$ y la de $j$ será $\gamma_{j i} f_{i} f_{j}^{2}$.

Matemáticamente, la dinámica puede expresarse a través de un sistema de $n$ ecuaciones diferenciales de la siguiente forma (López y Sanjuán, 2001)

$$
\frac{\partial f_{i}}{\partial t}=\alpha_{i} f_{i}\left(\beta_{i}-f_{i}\right)-\sum_{i \neq j} \gamma_{i j} f_{i} f_{j},
$$

donde:

$\alpha_{i}$ : tasa de crecimiento de cada sitio que mide la capacidad de dicho sitio para crecer.

$\beta_{i}$ : máxima capacidad del sitio que está relacionada con el valor de saturación de $f_{i}$

$\gamma_{i j}$ : fuerza de la competencia entre los sitios $i$ y $j$.

Los valores de los parámetros son tales que $\alpha_{i}>0,0 \leq \beta_{i} \leq 1$ y $\gamma_{i j} \geq 0$.

Valores crecientes del parámetro $\alpha_{i}$ implican un desarrollo más rápido del sitio $i$ en el mercado. En Internet los sitios que tienen una mayor tasa de crecimiento son aquellos que ofrecen contenidos sumamente interesantes que se actualizan de manera muy frecuente, vinculándose este parámetro con la calidad de los contenidos del sitio correspondiente (López y Sanjuán, 2001).

Por su lado, el parámetro $\beta_{i}$ que determina la máxima capacidad de un sitio está relacionado con el número de conexiones simultáneas que puede mantener, es decir, con la potencia de su servidor. Para simplificar el modelo se puede suponer que los sitios pueden actualizar su hardware y software lo suficientemente rápido como para nunca ser sorprendidos por problemas de congestión de tráfico en la Red; o dicho de otro modo, los sitios se comportan como si tuvieran una capacidad máxima de 1. (López y Sanjuán, 2001).

El parámetro $\gamma_{i j}$, en el que se materializa la tasa de competencia, puede considerarse como una medida del grado de incompatibilidad entre los sitios; lo que supone que si dos sitios se encuentran compitiendo con fuerza los clientes pueden visitar uno u otro, pero nunca los dos. Esto guarda relación con la similitud de los contenidos y servicios ofrecidos por los sitios, ya que no es REDMARKA - Revista Académica de Marketing Aplicado - CIECID - Universidad de A Coruña 
habitual que un cliente visite dos sitios que tienen los mismos contenidos y ofrecen los mismos servicios.

Con este planteamiento es factible poner de relieve que un sitio puede variar su nivel de competencia modificando sus contenidos, servicios, productos, precios, etc. Así, se va a considerar la hipótesis de que la tasa de competencia entre dos sitios es simétrica, esto es, $\gamma_{i j}=\gamma_{j i}$ (aunque esta hipótesis no se cumple en ciertos tipos de mercado es razonable pensar que en situaciones normales puede mantenerse). Nótese que en esta formulación del modelo sólo se considera el caso en que el coeficiente de competencia es positivo, lo que se traduce en que el término de interacción es siempre negativo (Choi, 1997). Con este argumento siempre existe una cierta tensión competitiva en las relaciones de los participantes aun cuando sean posibles estrategias de colaboración.

Aunque pudiera parecer que la definición de los parámetros sea abstracta, lo que resulta interesante no es tanto como pueden medirse para un sitio web real, sino su influencia sobre la fracción de clientes del sitio y sobre la evolución de la estructura del mercado en cuestión.

El sistema de ecuaciones que determina la dinámica no lineal de los visitantes de los sitios web es funcionalmente similar al descrito a través de las ecuaciones de presa-depredador en ecología ${ }^{3}$. De hecho, uno de los resultados más sorprendente (Maurer y Huberman, 2000) es la aparición repentina de un ganador que se lleva todo el mercado bajo condiciones de fuerte competencia y que captura a la mayoría de los visitantes, al menos en una configuración simétrica idealizada (este fenómeno que ha sido observado empíricamente por Adamic y Huberman (2000) en un estudio de mercados en la Red).

En algunos mercados el análisis del modelo puede ser realizado para el caso más general de $n$ sitios compitiendo. En otros casos es necesario hacer una hipótesis más restrictiva que supone reducir el análisis a un segmento del mercado que tenga sólo tres competidores. Esta restricción puede interpretarse 
como la derivada de la modelización de un segmento del mercado tomando sólo los tres competidores principales, considerando que el resto no interfieren en la dinámica de aquellos. Además, esta formulación representa el caso más sencillo donde se presentan simultáneamente estrategias de colaboración y competencia. En esta situación las ecuaciones podrían expresarse a través del siguiente sistema de ecuaciones diferenciales no lineales, donde se ha realizado el supuesto de que $\beta_{i}=1$ :

$$
\begin{aligned}
& f_{1}^{\&}=f_{1}\left(\alpha_{1}-\alpha_{1} f_{1}-\gamma_{12} f_{2}-\gamma_{13} f_{3}\right) \\
& f_{2}^{\&}=f_{2}\left(\alpha_{2}-\alpha_{2} f_{2}-\gamma_{21} f_{1}-\gamma_{23} f_{3}\right) \\
& f_{3}^{\&}=f_{3}\left(\alpha_{3}-\alpha_{3} f_{3}-\gamma_{31} f_{1}-\gamma_{32} f_{2}\right)
\end{aligned}
$$

Para comprender mejor las predicciones del modelo es necesario realizar el análisis de estabilidad de los equilibrios cuando se presentan distintas tasas de crecimiento y competencia para los distintos sitios (Maurer y Huberman (2000) han profundizado sobre los comportamientos en el caso de mercados en condiciones de simetría completa $\left.{ }^{4}\right)$.

Con carácter previo al análisis hay que definir con claridad ciertas premisas (López y Sanjuán, 2001):

- Se dice que el sitio $i$ tiene una fuerte competencia frente al sitio $j$ cuando $\gamma_{i j}>\alpha_{i}$.

- Se considera que un sitio $j$ tiene baja competencia con un sitio $i$ cuando $\gamma_{i j}<\alpha_{i}$.

- Un conjunto de sitios forman una colaboración cuando están en condiciones de competencia débil entre ellos, pero presentan tasas de competencia fuertes frente al resto de rivales.

- Se dice que el sitio $i$ es ganador cuando $f_{i}=1$ y $f_{j}=0$ para todo $i \neq j$. Asimismo, un grupo de sitios colaboradores gana si todos cumplen que $f_{i}>0$, y para el resto de los sitios $f_{i}=0$.

Los ocho puntos fijos del sistema así como los resultados de su análisis de estabilidad se ofrecen a continuación (López y Sanjuán, 2001):

1. La solución trivial $P_{0}=(0,0,0)$, que supone que los tres sitios desaparecen del mercado, es siempre inestable. 
2. Los puntos fijos $P_{1}=(1,0,0), P_{2}=(0,1,0)$ y $P_{3}=(0,0,1)$ corresponden a una situación de un único ganador. El análisis de estabilidad revela que el sitio $i$ ganará siempre que mantenga una fuerte competencia con el resto de los sitios con independencia de que éstos últimos puedan o no colaborar entre sí. Un ejemplo de una situación de este es el que aparece en el gráfico 1.

\section{-GRÁFICO 1-}

3. $P_{12}=\left(\frac{\alpha_{1} \alpha_{2}-\alpha_{2} \gamma_{12}}{\alpha_{1} \alpha_{2}-\gamma_{12}^{2}}, \frac{\alpha_{1} \alpha_{2}-\alpha_{2} \gamma_{12}}{\alpha_{1} \alpha_{2}-\gamma_{12}^{2}}, 0\right), P_{13}=\left(\frac{\alpha_{1} \alpha_{3}-\alpha_{3} \gamma_{13}}{\alpha_{1} \alpha_{3}-\gamma_{13}^{2}}, 0, \frac{\alpha_{1} \alpha_{3}-\alpha_{3} \gamma_{13}}{\alpha_{1} \alpha_{3}-\gamma_{13}^{2}}\right) \quad \mathrm{y}$ $P_{23}=\left(0, \frac{\alpha_{2} \alpha_{3}-\alpha_{2} \gamma_{23}}{\alpha_{2} \alpha_{3}-\gamma_{23}^{2}}, \frac{\alpha_{2} \alpha_{3}-\alpha_{2} \gamma_{23}}{\alpha_{2} \alpha_{3}-\gamma_{23}^{2}}\right)$ corresponden a situaciones donde gana la colaboración entre dos sitios que hace desaparecer al tercero, como se representa en el gráfico 2 (para los mismos valores de los parámetros que en el gráfico 1 excepto que ahora el grado de colaboración entre los sitios 1 y 2 es superior).

\section{-GRÁFICO 2-}

Una conclusión del análisis de estabilidad de estos equilibrios es que los puntos fijos $P_{3}$ y $P_{12}$ pueden coexistir bajo condiciones competitivas particulares. Sin embargo, el punto $P_{12}$ no es compatible con un el postulado el 'ganador se lleva todo', lo que prueba que en condiciones de competencia asimétrica esta circunstancia tiende a desaparecer (los puntos fijos $P_{13}$ y $P_{23}$ son similares pero con sus respectivas constantes).

4. También se establecen los puntos fijos válidos cuando los tres sitios coexisten: $P_{123}=\left(f_{1}^{0}, f_{2}^{0}, f_{3}^{0}\right)$. Aquí $f_{1}^{0}, f_{2}^{0}, f_{3}^{0}$ son las soluciones del siguiente sistema de ecuaciones lineales:

$$
\left\{\begin{array}{l}
\alpha_{1}=\alpha_{1} f_{1}^{0}+\gamma_{12} f_{2}^{0}+\gamma_{13} f_{3}^{0} \\
\alpha_{2}=\alpha_{2} f_{2}^{0}+\gamma_{12} f_{1}^{0}+\gamma_{23} f_{3}^{0} \\
\alpha_{3}=\alpha_{3} f_{3}^{0}+\gamma_{13} f_{1}^{0}+\gamma_{23} f_{2}^{0}
\end{array}\right.
$$

Para la obtención de los autovalores es necesario realizar supuestos muy restrictivos como la simetría completa en las tasas de competencia y crecimiento de los sitios, lo que ya se ha realizado con anterioridad; pero 
este punto sólo es estable bajo condiciones de competencia débil entre los sitios.

\section{TIPOS DE MERCADO}

Con el conocimiento de las particularidades del modelo y su dinámica, en lo que sigue se realiza una tipología de los mercados sobre la base del sistema descrito.

1. Mercados de decantación.

En estos mercados las condiciones de competencia son intensas, estando caracterizados por situaciones 'el ganador se lleva todo', esto es, presentan un sitio claramente ganador. Por ello en la literatura es frecuente que se use el término decantación, ya que después de una competencia inicial tienden a "decantarse" a favor de uno de los sitios que acaba haciéndose con todo el mercado (Gual y Ricart, 2001). Además, en estos mercados hay rendimientos crecientes conocidos como externalidades de red o economías de escala del lado de la demanda.

Las redes como Internet presentan una característica económica fundamental: el valor de conectarse a ella depende de cuántas personas estén ya conectadas. Son economías de escala del lado de la demanda que pueden derivar, en su forma más extrema, en un mercado donde un competidor se convierte en la única oferta posible. Estas dinámicas están impulsadas por el interés de los usuarios en seleccionar la tecnología que va a prevalecer al final; en suma, por el efecto de red o las externalidades de red (Shapiro y Varian, 1999).

Una característica significativa de las economías de escala del lado de la demanda es que, al contrario de las del lado de la oferta, no desaparecen cuando el mercado crece lo suficiente: el uso generalizado de la red justifica su utilización. Pero además se produce el fenómeno de las expectativas 
cumplidas, de tal forma que el éxito y el fracaso se deben tanto por la suerte y las expectativas de los consumidores como por el valor del producto.

No obstante, los efectos de red no funcionan de forma tan rápida o de manera tan predecible. Muchas veces se llegan a resultados bastante equilibrados tras duros y largos enfrentamientos. El estado inicial combinado con acontecimientos aleatorios o fluctuaciones actúan para empujar la dinámica dentro del dominio de uno de esos estados asintóticos y, por tanto, para seleccionar la estructura que el sistema adoptará finalmente. En este sentido, Arthur (1989) señala que la presencia de acontecimientos históricos de carácter fortuito pueden conducir al sistema a un resultado completamente distinto del que se hubiera producido en un escenario histórico diferente.

Por consiguiente, aunque esta dinámica autocatalítica da lugar a poderosos monopolios, el liderazgo del pionero no siempre es definitivo. Esto se manifestó en el éxito de Microsoft frente al first-mover Netscape en el mercado de los navegadores como relatan Evans y Wurster (1997). Precisamente, Porter (2001) concluye que los efectos de Red son importantes para llegar a una situación en la cual el ganador se lleve todo el mercado, pero es necesario que le pertenezcan en su totalidad. Además, en un entorno abierto como Internet, con sus estándares y protocolos comunes, es complicado para un solo sitio capturar los beneficios del efecto de Red.

2. Mercados de productos diferenciados.

En este mercado los competidores se encuentran bajo condiciones de competencia débil. De este modo, los distintos sitos web presentan poca incompatibilidad en sus contenidos ${ }^{5}$. Por tanto, ya que los contenidos de los sitios son diferentes se puede denominar a este mercado de productos diferenciados.

En estos mercados tiene lugar un cambio de la competencia por todo el mercado a una competencia por la cuota de mercado. Los oligopolios industriales de los sectores del automóvil, el acero, el aluminio, el petróleo, 
entre otros, presentaban este rasgo. Los intentos del líder del sector de capturar cuota a costa de los más pequeños provocaba a menudo fuertes respuestas al intentar las empresas pequeñas evitar que el uso de su capacidad decayera, impidiendo que el líder consiguiera una posición dominante. Las empresas podían perder competitividad y ser adquiridas o liquidarse, pero por lo general, había pocos cambios en las cuotas de mercado y la norma eran los oligopolios en lugar de los monopolios (Shapiro y Varian, 1999). Esto se refuerza por el hecho de que alcanzado un cierto tamaño a las empresas les resultaba difícil crecer debido a la complejidad creciente de controlar una empresa grande. Así, mientras que estas grandes empresas tenían que hacer frente a costes elevados las empresas pequeñas más flexibles encontraban nichos rentables.

Porter (2001) aprecia que a medida que va aumentando la penetración de un sitio web su oferta resulta menos adaptada a las necesidades del cliente. De esta forma, incluso si un sitio tiene capacidad para controlar un efecto de Red se llega a un punto de retornos decrecientes una vez alcanzada una masa crítica de consumidores. Esta situación presenta similitudes con el caso de que todos los sitios web controlan una fracción del mercado parecida que depende no sólo del valor particular de las tasas de competencia y crecimiento, sino también de las condiciones iniciales de los sitos. De hecho, bajo tasas de competición bajas los sitios son capaces de evolucionar siguiendo sus propias dinámicas sin ser interferidas por cualquier otro rival.

\section{Mercados mixtos: complementores.}

Aquí se presenta la peculiaridad de que algunos de los competidores colaboran entre sí mientras mantienen una fuerte competencia con el resto de los sitios rivales.

Los principales factores determinantes para decidir el grupo ganador son:

- La clase de mercado, esto es, el conjunto de parámetros de las ecuaciones que representan el sistema. 
- Las condiciones iniciales de los competidores que representan toda la historia pasada del mercado, si el sitio ya existe; o bien el mayor o menor éxito conseguido con la campaña de marketing si el sitio no existía con anterioridad.

En la caracterización de este tipo de mercados mixtos se permiten relaciones más complejas y diversas que la mera competencia o cooperación. Aparecen estrategias a medio camino entre ambas que Nalebuff y Brandenburger (1996) llaman estrategias de coopetición. A los participantes convencionales del mercado (clientes, proveedores y competidores) estos autores incorporan una nueva figura: los "complementores"6. Un sitio complementor podría definirse como aquel que aumenta la calidad o atractivo de otro sitio o, en los mismos términos, ve aumentado su atractivo debido a la existencia del otro.

No obstante, importa precisar que el término complementor es distinto al de aliado o asociado. Bajo un punto de vista estratégico, los términos asociado y aliado son demasiado restrictivos ya que no captan necesariamente la naturaleza integral de la relación de negocios (Nalebuff y Brandenburger, 1996). Una relación que, como se verá más abajo, intrínsecamente plantea ciertas tensiones. Por ello, López y Sanjuán (2001) definen la colaboración entre complementores como baja competencia y no como cooperación: dicho de otro modo, es una estrategia entre la competencia y colaboración.

Cuando se buscan complementores es aconsejable ofrecer interconexión. Es más, se debe reflejar la fortaleza del sitio que los ofrece ajustando los límites necesarios para reducir el riesgo de posibles pérdidas de control de la Red. En este contexto, jugarían un papel importante los enlaces (links) que conectan distintos sitios web con información o contenidos alternativos o diversos, derivándose un nivel de competencia o incompatibilidad entre los sitios bastante bajo. Con todo, el conjunto de sitios complementores se encuentra en condiciones de colaboración (baja competencia) entre ellos y, sin embargo, en fuerte competencia frente al resto de los rivales. 


\section{RECOMENDACIONES ESTRATÉGICAS EN FUNCIÓN DEL TIPO DE WEB SITE Y DE MERCADO}

Los comportamientos estratégicos sugeridos por el modelo en función del tamaño del sitio web dependen también del tipo de mercado en particular.

A la hora de aprovechar los resultados del modelo para definir la estrategia adecuada para cada sitio conviene enfatizar que estas conclusiones se obtienen a partir de un modelo que no representa una situación completamente real. Aun así, muchas características y comportamientos reales son explicados a partir de este modelo, dando lugar a una descripción coherente de los principales mecanismos que influyen sobre la competencia entre sitios web.

1. Estrategias para el sitio más fuerte.

Se dice que un sitio es el más fuerte cuando parte de las condiciones más favorables tomando como referencia su evolución en el mercado. Caso de ser nuevo, el referente es el éxito que se desprende de la campaña de promoción inicial. Esta última situación puede abordarse desde ópticas distintas:

a) Que se decida a colaborar con otros sitios, en lo que se ha definido más arriba mercado de productos diferenciados. El único punto estable bajo condiciones de simetría completa es (Sanjuán y López, 2001):

$$
f_{i}=\frac{\alpha}{\alpha+(n-1) \gamma},
$$

De forma que todos los sitios que compitan conseguirán la misma fracción del mercado con independencia de su condición inicial (en el caso general de $n$ sitios).

b) Que se encuentre en un mercado mixto, lo que puede plantear la posibilidad de realizar una colaboración con otro sitio compitiendo con un tercer rival. El modelo predice que bajo condiciones de simetría completa los únicos puntos estables son $P_{3}$ y $P_{12}$. $Y$ dado 
que los sitios que colaboran son los más fuertes es muy probable que el mercado converja al punto fijo $P_{12}$, implicando que los sitios 1 y 2 se encontrarán finalmente en la misma situación.

c) Que el sitio más fuerte se encuentre en un mercado de decantación y compita con los otros. El punto fijo de convergencia será aquel que hace que el sitio con la condición inicial más alta gane controlando la totalidad del mercado y consiguiendo que sus rivales desaparezcan a largo plazo.

Por todo esto, resulta obvio que el modelo predice que el sitio más fuerte está interesado en hacer que el mercado sea lo más competitivo posible.

Un ejemplo de liderazgo en la Red, aunque esta vez conseguido por un recién llegado, es el de Google.com. Los motores de búsqueda más populares como AltaVista o Inktomi habían dominado el mercado durante mucho tiempo antes de su llegada en 1997. Sin embargo, en menos de tres años Google se convirtió en el nodo más grande y el motor de búsqueda más visitado. El detonante que originó la exacerbada competencia que impulsó a Google al éxito fue un estudio realizado por Steve Lawrence y Lee Giles en el Instituto de Investigación NEC en Princeton. Según este estudio, todos los motores de búsqueda (Google incluido) tan sólo cubrían en 1999 alrededor del 40 por ciento del total del tamaño de la Red. Esto convirtió el tamaño en algo importante y los motores empezaron a competir duramente por la dominancia del mercado y a considerar el tamaño como el objetivo fundamental de su negocio. En junio de 2001 Google alcanzó por primera vez la mágica cifra de 1 billón de documentos cubiertos frente a otros rivales como Altavista o alltheweb.com. Hasta ahora, Google mantiene el liderazgo, Altheweb.com le sigue y Altavista es el tercero con alrededor de 5500 millones de documentos (Barabási, 2002). Pero por otro lado, junto con el tamaño hay otro factor importante que explica el éxito de Google: el algoritmo que utiliza para hacer las búsquedas es mucho más potente que el que tenían sus competidores. En consecuencia, la calidad de su servicio es superior, es decir, que el parámetro 
$\alpha_{i}$ está por encima de los de sus rivales, lo que hizo muy difícil la competencia al resto de motores.

\section{Estrategias para sitios web medianos y pequeños.}

Si un sitio que no es el más fuerte entra en un mercado donde existe un bajo nivel de competencia (mercado de productos diferenciados) tiene la opción de competir con todos los sitios o bien mantener un nivel de competencia bajo respecto a todos ellos. Es evidente que el primer caso no es el adecuado puesto que el sitio no es el más fuerte. En el segundo caso, y bajo condiciones de simetría completa, el único punto estable es aquel que tiene a todos los sitios coexistiendo con la misma fracción de mercado, por lo que cuanto más débil sea el sitio con respecto al resto más interesante será este tipo de mercado.

Para mantener este bajo nivel de competencia la empresa seguirá una estrategia de diferenciación encaminada a disminuir la tendencia que existe en los mercados de información a homogeneizar el producto, dedicando así sus esfuerzos a destacar por algún aspecto valorado por el clientes que haga de la oferta algo único y diferente a lo que se ofrece habitualmente al mercado.

Porter (2001) señala que para sobrevivir en Internet las puntocom deberían encontrar sus propias estrategias distintivas más que intentar imitarse unas a otras o copiar el posicionamiento de empresas ya establecidas. En vez de competir solamente a base de la variable precio deberían concentrarse en la selección y diseño de productos y contenidos propios, el servicio, la imagen y otras áreas que les proporcionen una diferenciación real.

Duran-subastas.com, por ejemplo, persigue la diferenciación ofreciendo la posibilidad de adquirir obras de arte a través de Internet a una base de clientes muy determinada y dispersa. Este tipo de productos muy diferenciados con targets fragmentados y difíciles de disponer en el mundo presencial son más susceptibles de tener éxito en Internet (Nueno et al., 2003). 
Por otra parte, cuando el mercado en el que incurre el sitio se encuentra en condiciones de competencia fuerte se le presentan las opciones de competir con todos o realizar un acuerdo de colaboración con otro rival. Los mercados de decantación presentan la característica de 'ganador se lleva todo', por lo que el sitio más fuerte hará que los demás desaparezcan y la estrategia de competencia será negativa. La segunda opción es la de colaborar con otro sitio según el análisis que sigue y que para simplificar vuelve al modelo de tres sitios. El sitio 1 que es el más pequeño entra en un segmento del mercado donde los sitios 2 y 3 compiten fuertemente, siendo el sitio 3 el más fuerte. Para realizar el acuerdo el sitio 1 tiene dos posibilidades: colaborar con el sitio 3 o con el sitio 2. El sitio 3 sería el socio más adecuado, aunque es mas que probable que el sitio 3 considere que un acuerdo con 1 no es lo que más le conviene.

La otra posibilidad en un mercado en condiciones de competencia fuerte es colaborar con el sitio que no es el más fuerte (el que hemos llamado sitio 2). Es probable que el sitio 2 esté dispuesto a colaborar con el sitio 1 para competir con el 3 porque una situación de mercado fuertemente competitivo con feedback positivo le llevaría a desaparecer. En un mercado mixto y asumiendo una configuración simétrica parcial $\left(\alpha_{1}=\alpha_{2}=\alpha_{3}=\alpha, \gamma_{13}=\gamma_{23}=\gamma_{c}, \gamma_{12}=\gamma_{a}\right)$ los únicos puntos fijos estables son $P_{12}$ y $P_{3}$. En esta situación, un factor muy importante a la hora de decidir la estrategia apropiada son las condiciones iniciales de los sitios. Así pues, el problema puede dividirse en dos partes (López y Sanjuán, 2001):

- La suma de las condiciones iniciales de los sitios 1 y 2 es mayor que la del sitio 3 (gráfico 3). En este caso parece probable que si el grado de colaboración es suficientemente fuerte el sitio 3 desaparecerá, incluso con tasas de competencia contra el grupo colaborador no muy fuertes. En estas condiciones la única probabilidad de subsistir para los sitios pequeños es permanecer juntos y colaborar.

\section{-GRÁFICO 3-}


- La suma de las condiciones iniciales del sitio 1 y el sitio 2 es menor que la del sitio 3 (gráfico 4). En estas condiciones aunque puede parecer lógico que el sitio más poderoso gane bajo cualquier circunstancia, el modelo predice que el grupo puede ganar al sitio 3 siempre y cuando el grado de colaboración sea alto y el grado de competencia con el sitio 3 sea fuerte pero no demasiado.

\section{-GRÁFICO 4-}

A resultados similares llegan Wang y Wu (2004) cuando introducen en el modelo valores negativos para la tasa de competencia, esto es, términos de interacción positivos en lo que denominan dinámicas de cooperación. Si la tasa de cooperación es lo suficientemente grande, la alianza entre los sitios pequeños tiene altas probabilidades de derrotar al sitio más poderoso sin necesidad de que se presenten los requisitos anteriormente expuestos acerca de la suma de condiciones iniciales.

En síntesis, para competir con éxito en un segmento del mercado de Internet donde ya existe un sitio poderoso, es más fácil ganar a este sitio buscando acuerdos de colaboración con complementores para enfrentarse al sitio más grande.

Si bien un sitio complementor es lo contrario de un sitio competidor, no se puede llamar aliado o asociado puesto que éste es un término más genérico que incluye clientes, proveedores y complementores. Además, como se ha citado, existen ciertas tensiones en la relación de los complementores ${ }^{7}$.

En la realidad de la Red puede observarse como las empresas se están desplazando hacia la coopetición como forma de estrategia alternativa a la mera competencia y que según se ha explicado permite una cooperación y competición simultánea entre los sitios. La competición posibilita el uso compartido de recursos en lugar de su duplicación o despliegue para contraatacar a la competencia (Sharma, 2002). Por ejemplo, algunos portales (Yahoo) utilizan motores de búsqueda de otros sitios (Google) que persiguen el mismo público objetivo. 
Con este movimiento hacia formas de cooperación y competición simultáneas, la atención gira de la lucha por el mercado hacia su desarrollo. En este sentido se están produciendo a escala internacional diversos tipos de colaboración, por ejemplo, en el ámbito de los sitios dedicados a los servicios de empleo, Infoempleo (España) ha firmado un acuerdo de colaboración con Jobsite.co.uk (Inglaterra), Trovojob.it (Italia), Jobscout24.de (Alemania) y Cadrempleoi.fr (Francia) para extender sus servicios de empleo a diferentes países europeos. De esta manera, se crea la red de empleo más extensa de Europa al tiempo que cada sitio afianza la posición que ocupa en su propio mercado. También los portales inmobiliarios líderes de Europa ${ }^{8}$ se han unido para crear la mayor plataforma on-line para la búsqueda de vivienda. A través de dicha vinculación se aprovechan del mercado potencial que permite el marco comercial comunitario al tiempo que aumentan las barreras de entrada que presenta un sector tan singular como el inmobiliario frente a los grandes grupos no europeos.

3. Estrategias para microsites y start-ups: Estrategias de segmentación y diversificación.

Aparte de la posibilidad de realizar acuerdos de colaboración con otro(s) sitio(s) para hacer frente común al líder, los sitios web que parten de una situación inicial más desfavorable y que pudieran tratarse de sitios de reciente creación (star-ups) o bien acaban de entrar en un nuevo segmento se le presentan otras oportunidades estratégicas.

Para sobrevivir en un mercado en fuerte competencia otra alternativa podría ser especializarse en atender a una parte del mercado en lugar de orientarse al conjunto del mismo. Para ello es necesario que el sitio se esfuerce en conocer las necesidades específicas de los clientes de su(s) segmento(s) y que trate de satisfacerlas como líder en costes o destacando por algún valor añadido en sus productos o contenidos. El éxito de esta estrategia estará tanto más asegurado cuando la fuerza de la competencia en dicho(s) segmento(s) no sea muy intensa. En la estrategia de segmentación el desafío consiste en encontrar una REDMARKA - Revista Académica de Marketing Aplicado - CIECID - Universidad de A Coruña 
propuesta de valor que distinga la web de otros rivales en Internet (Porter, 2001).

Otra alternativa para los sitios web pequeños o de reciente creación es entrar en los programas de afiliados que los portales suelen promover dentro de sus estrategias de crecimiento y diversificación (Ansoff, 1957). En numerosas ocasiones, detrás de los programas de afiliación se encuentra una estrategia de diversificación intentando el(los) sitio(s) afiliados alcanzar distintos segmentos con necesidades diferentes ofreciendo información y recursos de especial interés para esos segmentos específicos. Ello permite competir con los portales horizontales líderes que aportan una amplia variedad de materiales y servicios y que captan mucho público.

El modelo de dinámica competitiva que se estudia en el presente artículo también describe comportamientos que se pueden englobar en este tipo de estrategias y que parecen funcionar bastante bien para el modelo, y posiblemente también en Internet. A continuación se van a mostrar dos estrategias de este tipo descritas por el modelo.

- La primera estrategia se desarrolla en un segmento del mercado de Internet que está controlado por dos sitios que podrían denominarse 1 y 3 . El sitio 3 es el más importante contando con un 65\% del mercado, mientras que el sitio 1 sólo posee el 45\%. Mientras se mantenga esta situación (y a no ser que el sitio 1 realice la inversión necesaria para superar la cuota del 65\% del sitio 3) el sitio 3 mantendrá un fuerte nivel de competencia con el objetivo de hacer desaparecer al sitio 1 . No obstante, el modelo predice que si el sitio 1 crea un nuevo sitio pequeño (sitio 2 con un 10\% del mercado) y se afilia con él, el acuerdo de colaboración puede acabar con el sitio 3.

En el gráfico 5 se muestra esta situación. El sitio 2 sólo compite ligeramente con el sitio 3, lo cual es bastante factible puesto que no es probable que éste se preocupe por la presencia del sitio 2 .

\section{-GRÁFICO 5-}

REDMARKA - Revista Académica de Marketing Aplicado - CIECID - Universidad de A Coruña 
Esto significa que para entrar en un segmento del mercado de Internet donde ya existe un sitio poderoso es más económico ganar a este sitio creando dos sitios afiliados que un solo sitio grande. De hecho, es conocido el éxito de los portales temáticos que colaboran en Internet.

Los programas de afiliación son una práctica frecuente entre los grandes portales que pretenden cubrir las temáticas más demandadas para competir frente a los portales horizontales líderes. El problema al que se enfrentan este tipo de portales horizontales o generales es que sólo un número muy reducido puede ser capaz de ofrecer un adecuado nivel de servicios y contenidos a un público tan diverso y, a la vez, ser viable organizacional y económicamente (García Gómez, 2001). Por ello, los que sobrevivan tendrán que haber incorporado en sus programas de filiaciones pequeños portales verticales que proporcionen información y servicios a los diversos sectores que pretendan alcanzar con la profundidad, calidad y personalización de contenidos y servicios adecuada. La otra tendencia que se apunta para estos portales horizontales es la especialización geográfica, temática o corporativa.

Un ejemplo en consonancia con esta estrategia lo tenemos en el programa de afiliación de Internet.com, un portal líder de noticias en tiempo real y recursos de información para los profesionales y usuarios expertos de la Red. Este portal ha venido realizando múltiples acuerdos con diversos portales temáticos con el objetivo de proporcionar una amplia gama de servicios a sus visitantes así como diversificar sus fuentes de ingresos. Algunos de estos portales temáticos son: EXP.com dedicado a proveer servicios y consejos de expertos, Planet-Intra.com que ofrece Intranets gratuitas para PYMES, Domainbank.net especializada en servicios para registros de dominios en la Red, MyHelpdesk.com que proporciona recursos de ayuda informática y MLX.com comunidad online para la búsqueda de apartamentos en venta y alquiler en Nueva York. 
- El segundo tipo de estrategia presenta variadas posibilidades e interesantes comportamientos. Uno de los ejemplos más interesantes es el del mercado donde sólo compiten intensamente dos sitios realmente fuertes que se pueden denominar 1 y 2 . El sitio 1 ostenta el 65\% del mercado, mientras que el sitio 2 posee el $60 \%$. Bajo estas condiciones entra en escena un tercer sitio pequeño con sólo un $10 \%$ de la fracción del mercado. Como este sitio parece bastante inofensivo los sitios 1 y 2 no se preocupan en exceso por él, manteniendo un bajo nivel de competencia con el sitio 3, por lo que los contenidos del sitio 3 presentan una baja incompatibilidad con los de los sitios 1 y 2. En esta situación los sitios 1 y 2 acabarán destruyéndose uno al otro dejando que el sitio 3 gane (gráfico 6 ).

\section{-GRÁFICO 6-}

Un ejemplo de esta estrategia de segmentación es la acometida por Ferretería Ortiz, establecimiento especializado en la venta de metalistería, accesorios de baño, herrajes y cerrajería, que se ha enfocado en un público muy específico: anticuarios y profesionales del mueble, la decoración y la construcción. Gracias al alcance mundial de Internet ha llegado a segmentos similares en áreas difíciles de alcanzar por los medios tradicionales (Rodríguez Ardura, 2002).

En otro orden, la estrategia de segmentación puede ser una opción recomendable para pequeñas empresas locales que cuentan con recursos limitados en las que Internet les ofrece la posibilidad de poner en práctica iniciativas inalcanzables por otros medios. Esta oportunidad fue aprovechada por Barrabés, una tienda especializada en artículos de esquí y montaña situada en un pequeño pueblo de los Pirineos. Barrabés entendió la creación de una tienda virtual (Barrabes.com) como la mejor opción para superar las limitaciones propias de la estacionalidad de las ventas, la lejanía de las grandes ciudades y la escasez de recursos, aprovechando su condición de expertos en los productos que venían ofreciendo de forma convencional para venderlos en todo el mundo (Rodríguez Ardura, 2002). 
Por tanto, un sitio especializado en un segmento concreto puede aportar la experiencia suficiente para demandar una prima en Internet, lo que le permite sobrevivir frente a los grandes gigantes que proveen productos de docenas de diferentes categorías. Las webs de subastas online sportsauction.com o prosportsmemorabilia.com se han especializado en objetos de culto de deportes pudiendo obtener hasta un 35 por ciento más por transacción que lo que un cliente estaría dispuesto a pagar a través de una web de subastas de carácter genérico como eBay o Yahoo. La prima se debe fundamentalmente a qué en estos sitios especializados es mucho más fácil localizar los productos de esta categoría específica y acceder a la prestación de otros servicios extra como la garantía.

En último lugar, es importante reseñar que un aspecto sintomático en el segmento de contenidos es cómo los grandes sitios están reaccionando con retraso al reto que supone Internet, mientras que son los start-ups los que obtienen mejores resultados en la generación constante de nuevas ofertas (Gual y Ricart, 2001). Esto se confirma al analizar las rentabilidades de algunas empresas en Internet permitiendo concluir que en muchas ocasiones los sitios que sobreviven son los que se especializan en pequeños nichos de mercado utilizando modelos híbridos y pioneros (Nueno, et al., 2003).

\section{UNA PROPUESTA DE MATRIZ ESTRATEGICA PARA SITIOS DE INTERNET: ESTUDIO DE CASOS}

Las recomendaciones estratégicas proporcionadas por el modelo y aludidas en los epígrafes anteriores pueden representarse gráficamente en una matriz que resuma toda la información proporcionada. Para su elaboración se han considerado dos dimensiones básicas a la hora de proponer la estrategia óptima: el tipo de mercado y el tamaño de sitio web.

\section{-TABLA 1-}

Con respecto al tipo de mercado, para su identificación se utiliza como rasgo definitorio el grado de competencia mantenido entre los diversos sitios web, lo REDMARKA - Revista Académica de Marketing Aplicado - CIECID - Universidad de A Coruña 
que da lugar a los tres tipos expuestos más arriba (de mayor a menor competencia): Mercado de Decantación, Mercado Mixto o de Complementores, y Mercado de Productos Diferenciados.

En lo que se refiere a la segunda dimensión, se consideran tres tamaños del sitio web en función de la cuota de mercado inicial que tienen: sitios grandes, sitios medianos y pequeños; y sitios de reciente creación (start-ups) y sitios pequeños que acaban de entrar en un nuevo segmento.

De acuerdo con la matriz, la estrategia recomendada para el sitio con la mayor cuota de mercado inicial, es la de competir, ya que el modelo predice que a largo plazo terminará controlando el mercado y haciendo que desaparezcan sus rivales. En definitiva, lo que se intenta con esta estrategia es que, con independencia del tipo de mercado en el que se encuentre inicialmente, se ponga en marcha el feedback positivo (proceso dinámico que favorece al más fuerte) que en su forma extrema puede derivar en un mercado que se decanta a favor de uno de los sitios.

Para sitios de tamaño mediano a pequeño la estrategia depende del tipo de mercado de partida. Cuando se encuentran en un mercado de productos diferenciados la estrategia recomendada es intentar diferenciar sus productos de los demás disponibles en el mercado; lo que pueden conseguir personalizando sus contenidos. Esta estrategia será tanto más recomendable cuanto más pequeño sea el sitio en relación con el resto ya que el modelo predice que un mercado de este tipo y en condiciones de simetría completa la dinámica conduce a los agentes participantes hacia un feliz punto medio.

$\mathrm{Si}$, por el contrario, la web se encuentra en un mercado en el que existe fuerte competencia, con el propósito de que la situación no se incline hacia el más fuerte el modelo recomienda que busque complementores que se encuentren en una situación menos privilegiada que el más fuerte para coopetir con ellos y esperar a que el líder cometa algún error. Entonces, la recomendación que se deriva del análisis del modelo es que en un mercado con economías de escala 
del lado de la demanda es necesario buscar socios coopetidores. Conseguir y escoger colaboradores es un aspecto estratégico básico en la Economía de Redes (Shapiro y Varian, 1999).

Por último, se estudia el caso de sitios pequeños de reciente creación o que acaban de entrar en un nuevo segmento. Un caso de este tipo puede presentarse cuando dos sitios grandes con unas condiciones iniciales similares compiten fuertemente entre sí en un mercado con feedback positivo. En estas circunstancias al que parte de la menor condición inicial puede convenirle más que intentar expandir su cuota de mercado hasta superar la de su rival (lo que puede resultarle muy caro en términos de costes de promoción), diversificar sus contenidos creando nuevos pequeños sitios que ofrezcan información y recursos de especial interés para distintos segmentos con necesidades específicas y afiliarse con ellos. El modelo pronostica que bajo determinadas condiciones el socio principal o creador junto con sus pequeños sitios afiliados puede derrotar al rival más fuerte.

Otra posibilidad es que en ese mercado de fuerte competencia entre rivales poderosos similares entre en juego un sitio pequeño pero fuertemente concentrado en un segmento donde no existe apenas competencia y que seguramente se trate de un segmento muy nuevo para esperar a que los sitios grandes se destruyan o, al menos, pierdan su oportunidad de liderar también este nuevo segmento, pudiendo abrirse de forma paulatina a otros segmentos.

\section{CONCLUSIONES}

En el incierto y cambiante mundo de Internet el éxito e incluso la supervivencia para los sitios web se encuentra lejos de estar asegurada. El mercado de Internet presenta ciertas características y peculiaridades genuinas de las que se derivan importantes implicaciones estratégicas para sus participantes.

Entre las principales características de Internet destacan la naturaleza decreciente de sus costes de computación y comunicación, así como distintos tipos de rendimientos crecientes entre los que destacan los conocidos como REDMARKA - Revista Académica de Marketing Aplicado - CIECID - Universidad de A Coruña 
externalidades de red, que en su forma más extrema pueden derivar en la característica del 'ganador se lleva todo' el mercado.

Precisamente es la presencia de fuertes no-linealidades en este mercado la que hace especialmente apropiado para su estudio el uso de herramientas de dinámica no lineal como el modelo dinámico competitivo que se presenta en este trabajo. Dicho modelo no sólo captura algunos de los rasgos más importantes de Internet, como lo es la aparición de la característica del 'ganador se lleva todo' el mercado bajo condiciones de fuerte competencia entre sitios web y simetría completa, sino que, además, bajo condiciones más generales sugiere una serie de recomendaciones estratégicas alternativas a la mera competición y propias de los mercados mixtos, donde algunos competidores colaboran entre sí mientras compiten con el resto. Aparecen así la coopetición y la segmentación como las mejores opciones para la supervivencia frente a los gigantes de Internet. Asimismo, estas estrategias se muestran en consonancia con las tendencias observadas en la realidad de Internet.

En resumen, a partir del análisis técnico y cualitativo del modelos se ofrecen las siguientes recomendaciones en función tanto del tamaño del sitio como del tipo de mercado en el que éste se encuentra. Si se trata del sitio más fuerte, el modelo aconseja que la mejor estrategia es la de conseguir un mercado con los niveles de competencia más altos posibles (un mercado de decantación) con independencia del tipo de mercado de partida. Si, por el contrario, el sitio es pequeño, la mejor salida sería llegar a acuerdos con otros sitios en lo que se ha llamado una estrategia de coopetición y esperar que el líder cometa algún error. Otra posibilidad para los sitios pequeños es la de personalizar sus contenidos, intentando diferenciar sus productos de los demás en un mercado de competencia monopolística. Por último, para los sitios de nueva creación (start-ups) o recién llegados al mercado, se recomiendan las estrategias de afiliación o interconexión con otros sitios que ofrezcan información y recursos de interés para segmentos distintos al suyo, accediendo así a segmentos especializados que de otra forma sería difícil alcanzar; o de lo contrario intentar 
encontrar un segmento del mercado donde no exista un fuerte nivel de competencia y que probablemente se trate de un segmento nuevo.

\section{NOTAS}

1 Por ejemplo, para determinar el momento óptimo de introducción de un producto en el mercado cuando entran en juego sucesivas generaciones tecnológicas (Mahajan y Muller, 1995)

${ }^{2}$ Hay que hacer notar que $\gamma_{i j}$ no es necesariamente igual a $\gamma_{j i}$.

${ }^{3}$ También conocidas como ecuaciones de Lotka-Volterra.

${ }^{4}$ Se entiende por simetría completa el caso en el que las tasas de crecimiento y de competencia de todos los sitios son iguales, esto es, $\alpha_{i}=\alpha$ y $\gamma_{i j}=\gamma$.

5 Baja incompatibilidad significa que los contenidos no son similares y no complementariedad de contenidos, lo que supondría que el parámetro $\gamma_{i j}$ fuera negativo y el término de competencia positivo. Un análisis del modelo que incorpora valores del parámetro de competencia negativos es el realizado por Wang y Wu (2004) sugiriendo estrategias de cooperación similares a las ofrecidas en el presente trabajo.

${ }^{6}$ Traducción del término inglés complementors.

${ }^{7}$ Esto justifica el hecho de que aunque los sitios colaboren, el parámetro $\gamma$ sigue siendo positivo aunque pequeño .

8 Seloger.com e Immostreet.com (Francia), Expocasa.com (España), Immostreet.ch (Suiza), Funda.nl (Holanda), Vebra.com (Reino Unido), Immowelt.com (Alemania), Casa.it (Italia), y Belgimmo.be (Bélgica)

\section{BIBLIOGRAFÍA}

- Aaker, D.A. (2002): "The Internet as Integrator. Fast Brand Building in SlowGrowth Markets", Strategy+Business, Third Quarter; http://www.strategy-business.com/media/pdf/02306.pdf

- Adamic, L.A. y Huberman, B.A. (2000): "The Nature of Markets on the World Wide Web", Quarterly Journal of Electronic Commerce 1, 1. pp. 5-12; 
http://www.parc.xerox.com/spl/groups/dynamics/abstracts/Ecommerce/webm arkets.html

- Ansoff, H. I. (1957): "Strategies for diversification", Harvard Business Review, September-October, pp. 113-124.

- Arthur, W.B. (1989): "Competing Technologies, Increasing Returns, and Lock-in by Historical Events", The Economics Journal, vol. 99, March, pp. 116-131.

- Arthur, W.B. (1996): "Increasing Retursn and the New World of Business", Harvard Business Review.

- Barabási, A.-L. (2002): Linked. The New Science of Networks, Perseus Publishing, Cambridge.

- Bass, F.M. (1969): "A New Product Growth Model for Consumer Durables", Management Science, vol. 15, January, pp. 215-227.

- Brandenburger, A. y Nalebuff, B. (1997): "Complementors in the Digital Economy", Oracle Alliance, Marzo-Abril; http://mayet.som.yale.edu/coopetition/news/oracle.html.

- Choi, C.-H. (1997): "Generalizations of the Lotka-Volterra Population Ecology Model: Theory, Simulation, and Applications", Nonlinear Dynamics, Psychology, and Life Sciences, vol. 1, 4, pp. 263-273.

- Dutta, S. y Biren, B. (2001): "Business Transformation on the Internet, Results from the 2000 Study", European Management Journal, Vol. 19, № 5 , pp. 449-462.

- Evans, P.B. y Wurster, T. S. (1997): "Strategy and the New Economics of Information", Harvard Business Review, septiembre-octubre, pp. 71-82.

- García Gómez, J.C. (2001): "Portales de internet: coceptos, tipología básica y desarrollo", El profesional de la información, vol. 10, $n^{\text {os }} 7-8$, julio-agosto, pp. 4-13.

- García-Gutiérrez Fernández, C., Fernández Guadaño, J, García Villalobos, J.C. y Miranda García, M. (2004). "La red de información -INTERNET- de la sociedad de la comunicación y del conocimiento promueve la generalización de la participación en los procesos de toma de decisiones (en el establecimiento de los objetivos) en las organizaciones de producción", Ciriec-España, n 49, pp. 31-54. 
- Gual, J. y Ricart J.E. (2001): Estrategias Empresariales en Telecomunicaciones e Internet, Fundación Retevisión, Madrid.

- Griffith, V. (1999): "Tailored Marketing on the Internet. Does it really capture customers?", Strategy+Business, Fourth Quarter; http://www.strategy-business.com/media/pdf/99405.pdf.

- Guastello, S.J. (1992): "Population dynamics, and workforce productivity", en Michaels, M. (ed.) Proceedings of the annual chaos network conference, pp. 120-127, People Technologies, Urbana, IL.

- Hannan, M.T. y Freeman, J.H. (1977): "The population ecology of organizations", American Journal of Sociology, vol. 82, pp. 929-964.

- Hannan, M.T. y Freeman, J.H. (1988): "Density dependence in the growth of organizational populations", en Carroll, G.R. (ed.) Ecological models of organizations, Ballinger, Cambridge, MA.

- Katz, M.S. y Rothfeder, J. (2000): "Crossing the digital divide", Strategy+Business, First Quarter; http://www.strategy-business.com/media/pdf/001041.pdf

- Katz, M.L. y Shapiro, C. (2001): "Network Externalities, Competition, and Compatibility", American Economic Review, vol. 75, n³ 3, pp. 424-440.

- Lieberman, H.B. y Montgomery, D.B. (1988): "First Mover Advantages", Strategic Management Journal, Vol. 9, pp. 41-58.

- López, L. y Sanjuán, M.A.F. (2001): "Defining strategies to win in the Internet market", Physica A 301, pp. 512-534.

- McKnight, L.W., Vaaler P.M., Katz, R.L., (2001): Creative Destruction. Business Survival Strategies in the Global Internet Economy, The MIT Press, Cambridge.

- Mahajan, V. y , Muller, E. (1995): "Timing, Diffusion and Substitution of Successive Generations of Technological Innovations: The IBM Mainframe Case", Working Paper, Department of Marketing, The University of Texas at Austin

- Maurer S.M. y Huberman, A. (2000): "Competitive Dynamics of Web Sites", Xerox Palo Alto Research Center, arXiv:nlin.CD/0003041;

http://www.parc.xerox.com/spl/groups/dynamics/abstracts/Ecommerce/winne r.html

REDMARKA - Revista Académica de Marketing Aplicado - CIECID - Universidad de A Coruña 
- Nalebuff, B.J. y Brandenburger, A.M. (1996): Coopetition, Harvard Business School Press, Cambridge.

- Nieto de Alba, U. (1998): Historia del tiempo en Economía. Predicción, caos y complejidad, McGraw-Hill, Madrid.

- Nueno, J.L., Viscarri, J. Y Mora C. (2003): “¿Hay BTC en España?”, Documento de Investigación nº 499 de IESE, Universidad de Navarra.

- Ohmae, K. (2000): "The Godzilla Companies of the New Economy", Strategy+Business, First Quarter; http://www.strategy-business.com/media/pdf/00112.pdf

- Peters, B.G. y Hogwood, B.W. (1991): “Applying population ecology models to public organizations" en Perry, J.L. (ed.) Research in Public Administration, vol. 1, pp. 79-108.

- Porter, M. E. (2001): "Strategy and the Internet". Harvard Business Review, marzo, pp. 63-78.

- Rodríguez Ardura, I. (2002): Marketing.com y comercio electrónico en la sociedad de la información, Pirámide, Madrid.

- Shankar, V. y Bayus, B.L. (2003): "Network Effects and Competition: An Empirical Analysis of the Home Video Game Industry", Strategic Management Journal, vol. 24, nº 4, pp. 375-384.

- Shapiro, C. y Varian, H.R. (1999): Information rules: a strategic guide to the network economy, Harvard Business School Press.

- Sharma, A. (2002): "Trends in Internet-based business-to-business marketing", Industrial Marketing Management, 31, pp. 77-84.

- Wang, Y. y Wu, H. (2004): "Dynamics of a cooperation-competition model for the WWW market", Physica A 339, pp. 609-620. 


\section{GRÁFICOS}

GRÁFICO 1. Trayectorias temporales de las cuotas de mercado de tres sitios con una coalición derrotada (para los valores de los parámetros $\alpha_{1}=\alpha_{2}=\alpha_{3}=1, \gamma_{12}=\gamma_{21}=1.2$ y $\gamma_{13}=\gamma_{31}=\gamma_{23}=\gamma_{32}=2.5$, y condiciones iniciales $f_{1}=0.4, f_{2}=0.1, f_{3}=0.5$ ).

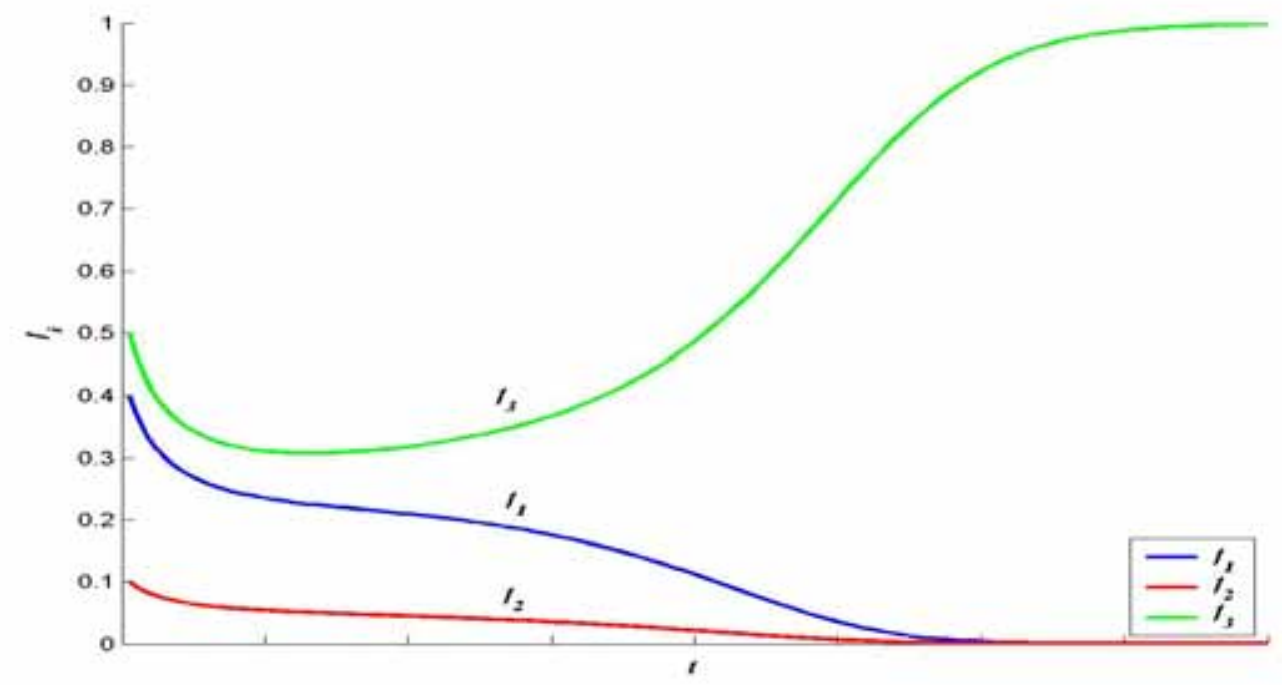


GRÁFICO 2. Trayectorias temporales de las cuotas de mercado de tres sitios con una coalición ganadora (para los valores de los parámetros $\alpha_{1}=\alpha_{2}=\alpha_{3}=1, \gamma_{12}=\gamma_{21}=0.3$ y $\gamma_{13}=\gamma_{31}=\gamma_{23}=\gamma_{32}=2.5$, siendo las condiciones iniciales $f_{1}=0.4, f_{2}=0.1, f_{3}=0.5$ ).

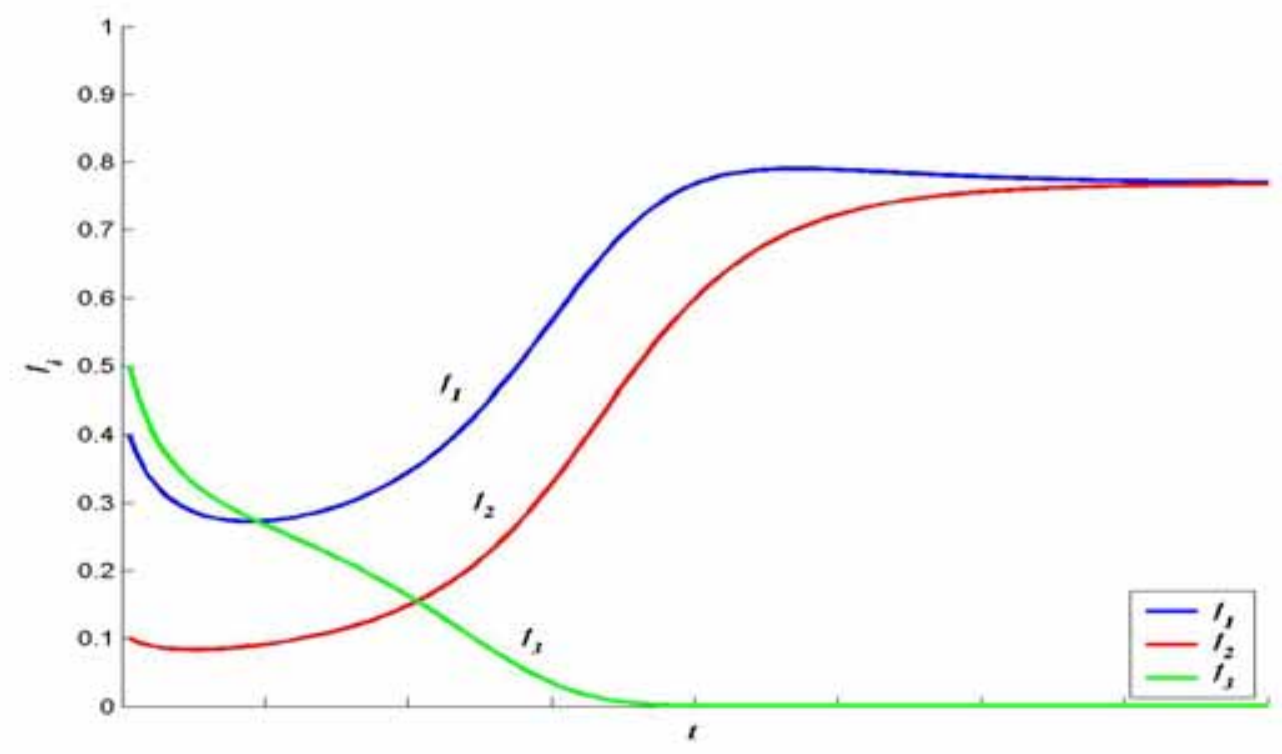


GRAFíco 3. Trayectorias temporales de las cuotas de mercado de tres sitios con una coalición ganadora de pequeñas webs, cuando la suma de las condiciones iniciales de éstas supera a la del sitio más fuerte (para los valores de los parámetros $\alpha_{1}=\alpha_{2}=\alpha_{3}=1, \gamma_{13}=\gamma_{23}=1.5, \gamma_{12}=0.5$, y condiciones iniciales $f_{1}=0.2, f_{2}=0.3, f_{3}=0.4$ ).

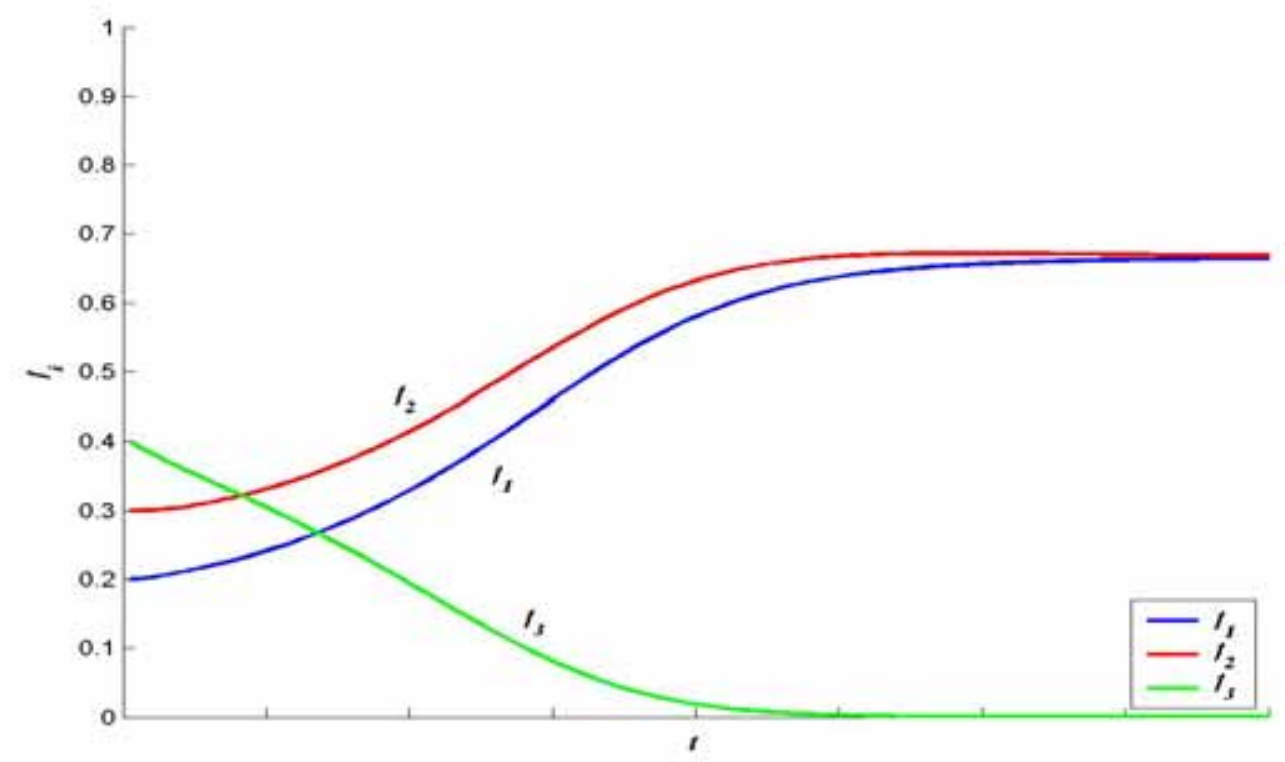


GRÁFICO 4. Trayectorias temporales de las cuotas de mercado de tres sitios con una coalición ganadora de pequeñas webs, cuando la suma de las condiciones iniciales de éstas es inferior a la del sitio más fuerte (para los

valores de los parámetros $\alpha_{1}=\alpha_{2}=\alpha_{3}=1, \gamma_{13}=\gamma_{23}=1.5, \gamma_{12}=0.4 \mathrm{y}$ condiciones iniciales $f_{1}=0.1, f_{2}=0.2, f_{3}=0.4$ ).

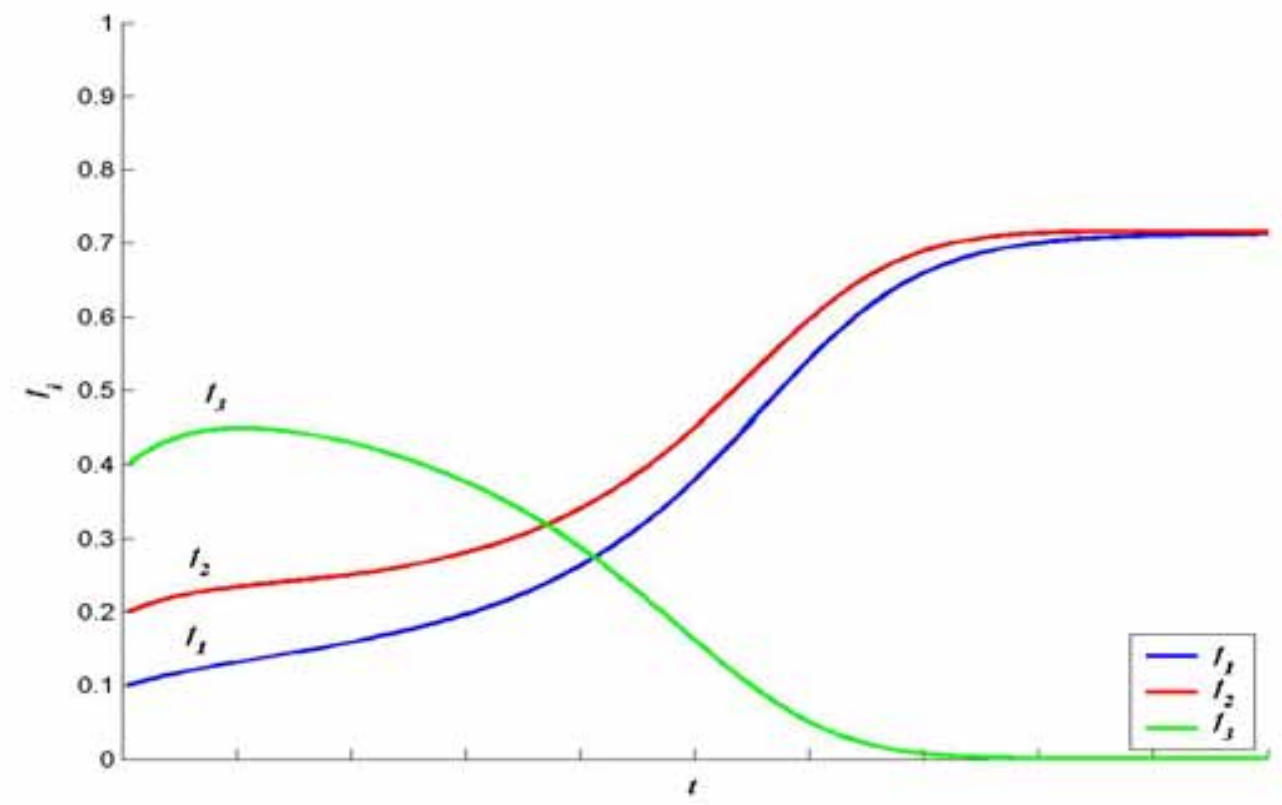


GRÁFICO 5. Trayectorias temporales de las cuotas de mercado de tres sitios en un mercado segmentado con una coalición ganadora (para el conjunto de parámetros $\alpha_{1}=\alpha_{2}=\alpha_{3}=1, \gamma_{13}=\gamma_{31}=2, \gamma_{12}=\gamma_{21}=0.3, \gamma_{23}=\gamma_{32}=1.2$. Las condiciones iniciales son $f_{1}=0.45, f_{2}=0.1, f_{3}=0.65$ ).

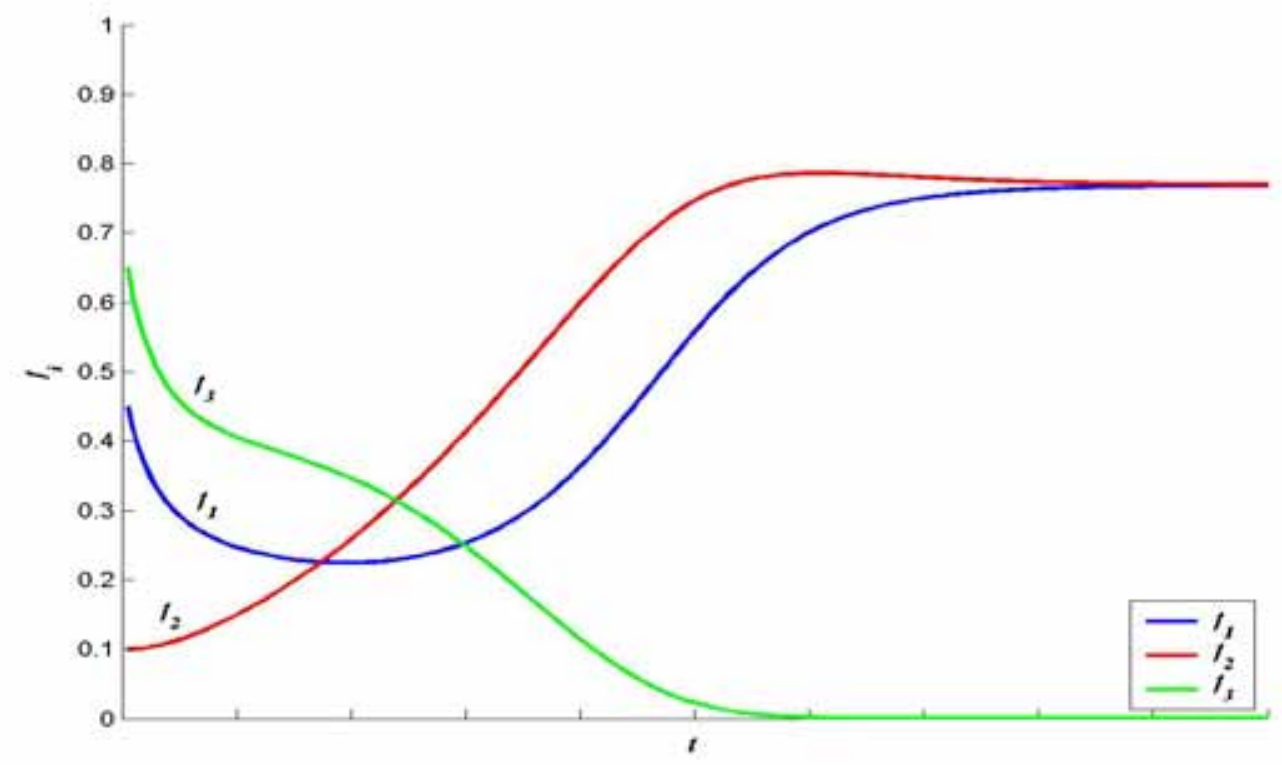


GRÁFICO 6. Trayectorias temporales de las cuotas de mercado de tres sitios en un mercado segmentado con un pequeño sitio vencedor (para el conjunto de parámetros $\alpha_{1}=\alpha_{2}=\alpha_{3}=1, \gamma_{21}=\gamma_{12}=2.5$, $\gamma_{31}=\gamma_{13}=1.1 \gamma_{23}=\gamma_{32}=1.2$, y condiciones iniciales $f_{1}=0.65, f_{2}=0.6, f_{3}=0.1$ ).

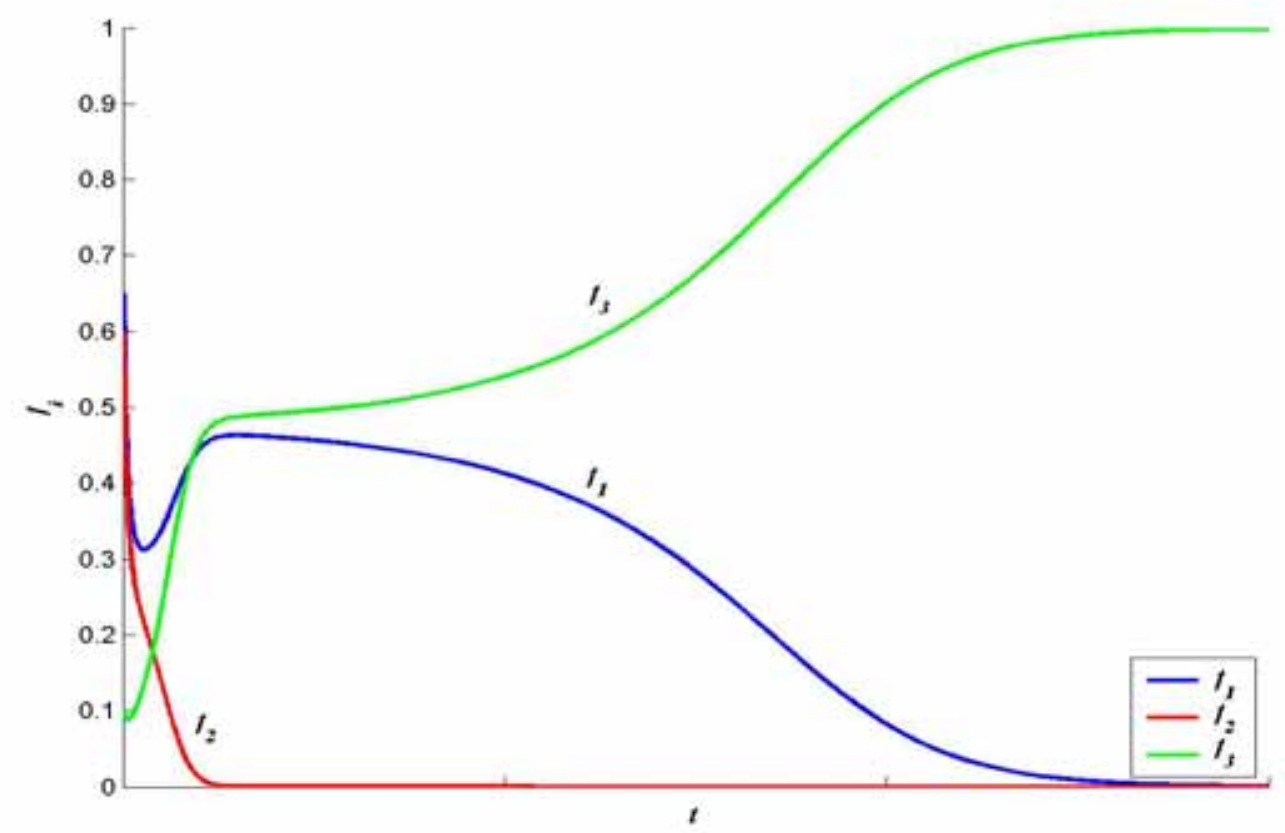


TABLA 1. Matriz de recomendaciones estratégicas para sitios web en función de su tamaño y del tipo de mercado en el que se encuentren.

\begin{tabular}{|c|c|c|c|}
\hline $\begin{array}{l}\text { Tamaño del sitio } \\
\text { Tipo de } \\
\text { mercado }\end{array}$ & Sitios grandes & $\begin{array}{c}\text { Sitios medianos y } \\
\text { pequeños }\end{array}$ & $\begin{array}{c}\text { Micro-web sites } \\
\text { y start-ups }\end{array}$ \\
\hline $\begin{array}{l}\text { Mercado de } \\
\text { Decantación }\end{array}$ & $\begin{array}{c}\text { LÍDER DE } \\
\text { RED }\end{array}$ & COOPETICIÓN & $\begin{array}{l}\text { SEGMENTACIÓ } \\
\text { PROGRAMAS } \\
\text { DE } \\
\text { AFILIACIÓN }\end{array}$ \\
\hline $\begin{array}{l}\text { Mercado } \\
\text { Mixto }\end{array}$ & COMPETIR & $\begin{array}{c}\text { COALICIÓN } \\
\text { DE } \\
\text { COMPLEMENTO } \\
\text { RES }\end{array}$ & $\begin{array}{l}\text { PORTALES } \\
\text { VERTICALES } \\
\text { INTERCONECT } \\
\text { ADOS }\end{array}$ \\
\hline $\begin{array}{c}\text { Mercado } \\
\text { Diferenciado }\end{array}$ & COMPETIR & $\begin{array}{c}\text { DIFERENCIACIÓ } \\
\mathbf{N}\end{array}$ & $\begin{array}{l}\text { DIFERENCIACI } \\
\text { SEGMENTACIÓ } \\
\text { S }\end{array}$ \\
\hline
\end{tabular}

Para citar este artículo:

García Villalobos, Juan Carlos - Mateos de Cabo, Ruth - López Fernández, Luis Fernández Sanjuán, Miguel Ángel (25-02-2009). ESTRATEGIAS EN INTERNET A PARTIR DE UN MODELO DE DINÁMICA COMPETITIVA.

REDMARKA - CIECID - Unidad de Investigación en Marketing Aplicado-Universidad de A Coruña

Año I, Número 1, V1, pp.39-82

ISSN 1852-2300

URL del Documento: http://www.cienciared.com.ar/ra/doc.php?n=1002 